\title{
Modified small bowel CT (MBCT) for evaluating sub-acute and chronic conditions affecting the small bowel: a low radiation-dose, unenhanced $\mathrm{CT}$ protocol
}

\author{
Ania Zofia Kielar • Hardy Tao $\cdot$ Seng Thipphavong
}

Received: 21 November 2009/Revised: 23 January 2011 /Accepted: 8 June 2011 /Published online: 1 July 2011

(C) European Society of Radiology 2011

\section{Introduction}

Modified Small Bowel CT (MBCT) is a low-dose CT method of evaluating chronic conditions affecting the small bowel. Due to ingestion of hyperdense oral contrast, there is resultant high contrast resolution between the bowel lumen, bowel wall and surroundings.

This technique can be used in follow-up of chronic conditions affecting the small bowel, including Crohn's disease, postoperative bowel leaks and recurrent small bowel obstructions due to strictures and small bowel luminal growths. MBCT is easy and comfortable for the patient to undergo, takes a very short time to acquire images and allows reconstruction of images in any plane by the radiologist during post-processing.

\section{Description of technique}

Compared with fluoroscopic small bowel enteroclysis or $\mathrm{CT}$ enteroclysis, MBCT is non-invasive and less timeconsuming for radiologists, since there is no need for

\section{Grant sponsors None}

A. Z. Kielar $\cdot$ H. Tao $\cdot$ S. Thipphavong

Faculty of Medicine, University of Ottawa,

Ottawa, ON, Canada

A. Z. Kielar $\cdot$ H. Tao $\cdot$ S. Thipphavong

Department of Diagnostic Imaging, University of Ottawa,

Ottawa, ON, Canada

\section{A. Z. Kielar $(\bowtie)$}

The Ottawa Hospital,

Civic Campus 1053 Carling Avenue,

Ottawa, ON, Canada

e-mail: aniakielar@gmail.com duodenal intubation or spot imaging at the time of image acquisition. It does not use intravenous dye and therefore is safer for the patient and less expensive than CT enterography or standard contrast-enhanced CT scans.

As part of the MBCT protocol, patients fast for 6 hours prior to the exam. No bowel preparation or nasogastric tube insertion is needed. An oral solution of $9 \%$ Telebrix-38 (meglumine ioxitalamate; Tyco healthcare, Quebec, Canada) is administered rather than 3\% used for most standard CT scans of the abdomen and pelvis. This oral contrast has a high attenuation (500 to $600 \mathrm{HU}$ ), increasing contrast resolution on this unenhanced CT protocol. Denser oral contrast cannot be used as it would cause beam hardening artifacts. Patients drink $300 \mathrm{ml}$ aliquots of $9 \%$ of oral contrast every 15 minutes over 60 minutes, for a total volume of $1200 \mathrm{ml}$. The isotonic nature of the $9 \%$ oral contrast gives better distension of the bowel lumen compared to water or other lower osmolal solutions, since there is physiologically less absorption of the isotonic solution by the small bowel. The hyperdense oral contrast is visible on scout images, thus when it is seen in the ascending colon, this indicates satisfactory opacification of the small bowel. A Noise Index of 28 is used with Auto-mA modulation on 16 or 64-slice scanners (GE Lightspeed, VCT LightSpeed (Germany)) compared to a typical noise index of 12-18 for a standard abdominopelvic CT scan. Image noise (GE Healthcare, noise index [NI]) is an operator-selected variable which alters the range of $\mathrm{mA}$ over which an automated tube current modulation varies during gantry rotation. In this way, one can produce a selected level of average noise in an image [1]. This higher noise index can be used in of the MBCT because there is inherent high contrast between the hyperdense oral contrast used in this CT protocol, and the bowel wall. The combination of high Noise Index and Auto-mA modulation reduces patient radiation dose. Remaining parameters include $120 \mathrm{kV}, \mathrm{mA}$ range of 100 to $240 \mathrm{~mA}$, a $0.5 \mathrm{sec}$ gantry 
rotation and a pitch of 1 , with slice reconstruction of 0.5 to $1.25 \mathrm{~mm}$, depending on the scanner used. Prone and supine scanning ensures opacification of all parts of the small bowel, but at the discretion of the radiologist, the prone scan can be omitted, particularly in follow-up studies. Images are viewed at a window of 800-900 HU and level of 200-300 HU.

Radiation dose from a supine acquisition with the above parameters have a dose length product (DLP) of 150 mGycm, which equates to approximately $2.55 \mathrm{mSv}$ ( range $1.57-5.23 \mathrm{mSv}$ ) This is calculated using the formula for estimated radiation exposure E $=$ DLP x 0.015 (abdominal conversion factor) [2]. Barium procedures such as SBFT and enteroclysis have been reported to have effective doses of $13.68 \pm 6.85 \mathrm{mSv}$ and $13.99 \pm 7.57 \mathrm{mSv}$ respectively [3]. CT enterography mean effective doses have also been reported at $9.58 \mathrm{mSv}$, with additional $1.13 \mathrm{mSv}$ for the placement of the nasojejunal tube [4].

Reconstructed thin slices are post-processed to produce maximum intensity projections (MIP), multiplanar reformats (MPR), and virtual endoscopy images, which have been shown to increase diagnostic confidence $[5,6]$. On average reconstructions take less than 5 minutes, but may require up to 20 minutes, depending on the case's complexity.

\section{Discussion}

With increasing concerns about patient radiation exposure from medical imaging, the development of low-radiation CT protocols is of value. This has been employed for renal stone evaluation, diagnosing suspected diverticulitis and for lung nodule follow-up [7, 8, 9]. The good spatial resolution, high contrast resolution, fast image acquisition and multi-planar reconstruction capabilities of computed tomography make this imaging modality very attractive for imaging the bowel as well. MRI and sonography are other imaging techniques that do not expose patients to ionizing radiation that can also be used to evaluate the bowel. Although MRI does not use radiation and has excellent contrast resolution, it is not as easily accessible, it requires the use of intravenous contrast, and the fairly long scan times can affect image quality because of intrinsic peristalsis of the small bowel [10].

Sonography can depict areas of bowel wall thickening and areas of inflammation; however, there are limitations, such as operator dependence, inter-observer agreement and even intra-observer agreement in the same patient during follow-up imaging. Additionally, detection and characterization of complex entero-enteric fistulas can be difficult [11]. Thus, we have developed a low-radiation dose, modified bowel CT (MBCT) enterography technique using hyperdense oral contrast, without intravenous contrast. MBCT can specifically evaluate small bowel while exposing the patient to less radiation compared to SBFT and CT enterography [4]. MBCT can characterize small bowelrelated abnormalities, such as hernias, acute versus chronic Crohn's disease, postoperative fistulas or bowel leaks and small bowel intraluminal masses including the site of chronic, low-grade bowel obstructions.

Applications

\section{Follow-up of Crohn's disease}

Active Crohn's disease on CT enterography is identified by increased mural attenuation, increased mural thickness, mural stratification and vasa recta hypervascularity $[12,13]$. On SBFT, there is luminal narrowing with irregularity, but differentiation from chronic disease is not always possible [14]. On MBCT, increased mural thickness, increased mesenteric fat attenuation and prominent vasa recta can be identified, indicating active disease (Fig. 1). MBCT imaging for Crohn's disease can delineate extraluminal complications such as fluid collections and enterocutaneous/enteroenteric fistulas. MBCT can also identify widening and fatty infiltration of the submucosal layer of the bowel due to chronic inflammation, consistent with the fat-halo sign [15]. Extra-enteric abnormalities associated with Crohn's disease, including gallstones, renal stones and sacroiliitis, can often be identified.

\section{Small bowel hernias}

Abdominal hernias are evaluated using MBCT, especially with provocation techniques such as Valsalva manoeuvres, to enhance visualisation of the area of herniation.

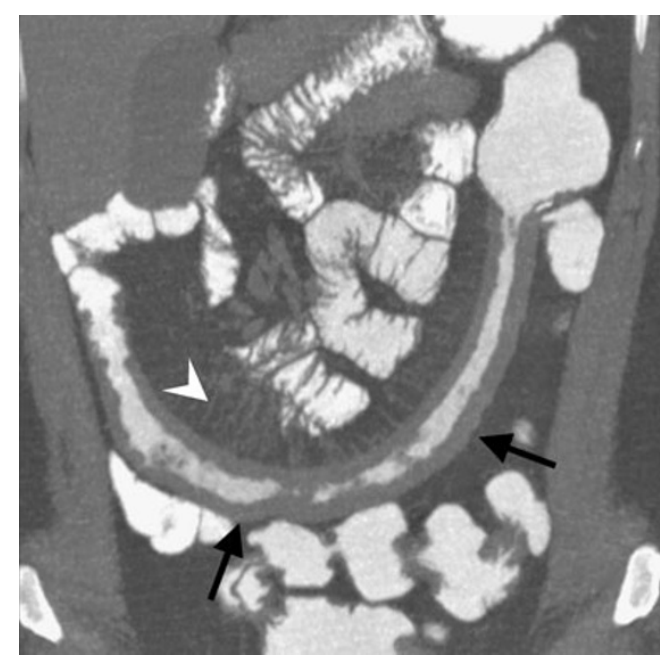

Fig. 1 A 38-year-old with Crohn's disease. There is mural thickening of the ileum (black arrows), increased mesenteric fat attenuation and prominence of the vasa recta (white arrowhead), all in keeping with active disease superimposed on previously documented chronic thickening of the ileum 


\section{Post-operative small bowel leaks/fistulas}

Compared with fluoroscopic techniques, MBCT is advantageous in the assessment of enteric fistulas because of its ability to define the cross-sectional location of the underlying disease (Fig. 2a, b).

\section{Chronic low-grade obstructions}

Modified bowel CT is useful in identifying chronic lowgrade obstructions, including the dilated segment and location of obstruction. Often, the diagnosis is that of adhesions, with abnormal tethering of small bowel loops and an associated distinct transition point indicating the site of obstruction. Strictures can also be characterised, including the length of bowel involvement and the severity of luminal narrowing.

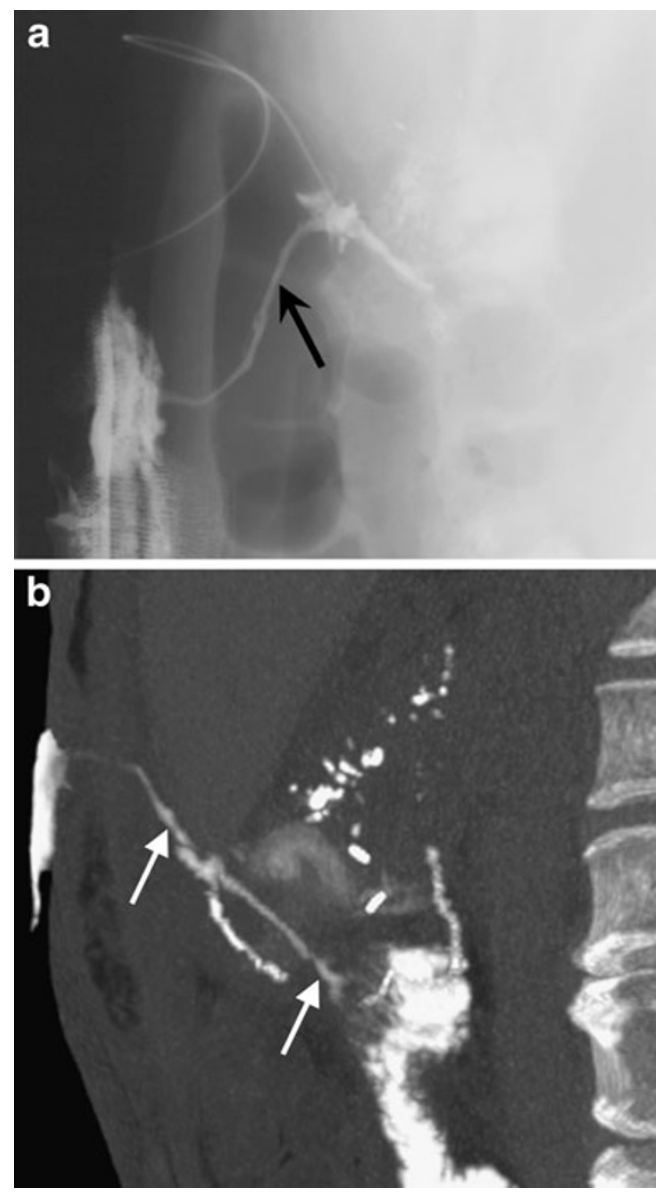

Fig. 2 A 46-year-old woman underwent Whipple's surgery for pancreatic cancer and presented with a draining sinus. A conventional fistulogram was performed demonstrating the fistula tract (black arrow) communicating with a jejunal loop, but further characterisation was difficult secondary to overlapping structures (a). MBCT demonstrates the complex nature of the fistula, with branching sinus tracts and two connections to the afferent jejunal loop (white arrows) (b)

\section{Small bowel tumours}

Small bowel neoplasms can be identified as a filling defect or an area of narrowing on MBCT. MBCT can depict areas of irregular luminal narrowing and shouldering of the margins, raising the suspicion of an underlying malignancy (Fig. 3a, b). Extrinsic masses, such as adenopathy and abnormal mesenteric masses, are also identifiable on MBCT.

\section{Limitations}

This technique is not recommended for evaluating patients with non-specific abdominal pain, unexplained weight loss or possible metastatic disease. As MBCT does not use intravenous contrast material, solid organs cannot be adequately evaluated. Differentiating a phlegmon from a mature abscess can be challenging without the use of intravenous contrast material. This technique should not be
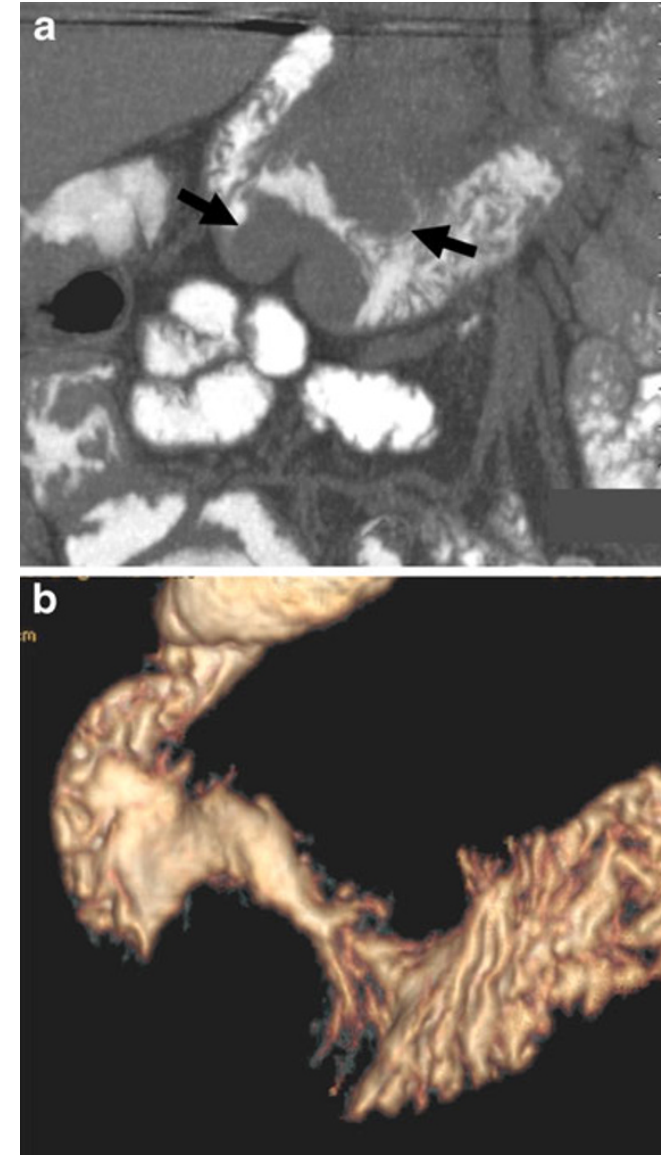

Fig. 3 A 94-year-old man with a mass suspected on standard CT underwent MBCT, which demonstrated a mass in the third part of the duodenum and luminal narrowing (a). Volume rendering demonstrates the narrowing of the bowel lumen and "shouldering" appearance of the mass (b). Endoscopy with biopsies confirmed an adenomatous polypoid lesion with foci of high-grade dysplasia 
used in acute GI bleeding since the hyperdense oral contrast will obscure visualisation of the luminal blood.

\section{Conclusion}

Amid increasing concerns about radiation exposure in patients, MBCT is a safe, simple and practical way to evaluate the small bowel specifically. This technique uses less radiation then $\mathrm{CTE}$ or SBFT, and images can be reconstructed in any plane, obviating SBFT limitations of overlapping bowel loops and reducing risks by eliminating intravenous contrast material administration. The goal of developing MBCT is to replace SBFT; a direct comparison of MBCT with SBFT, with surgical outcomes and imaging follow-up, is planned for evaluating specific small bowel abnormalities, including recurrent symptoms of established Crohn's disease and for the evaluation of hernias.

\section{References}

1. Kanal KM, Stewart BK, Kolokythas O, Shuman WP (2007) Impact of operator-selected image noise index and reconstruction slice thickness on patient radiation dose in 64-MDCT. AJR Am J Roentgenol 189(1):219-225

2. Huda W, Kent MO, Khorasani MR (2008) Converting dose-length product to effective dose at CT. Radiology 248:995-1003

3. Ruiz-Cruces R, Ruiz F, Perez-Martinez M, Lopez J, Tort Ausina I, de los Rios AD (2000) Patient dose from barium procedures. Br J Radiol 73:752-761

4. Wold PB, Fletcher JG, Johnson CD, Sandborn WJ (2003) Assessment of small bowel Crohn disease: noninvasive peroral CT enterography compared with other imaging methods and endoscopy-feasibility study. Radiology 229:275-281
5. Raptopoulos V, Schwartz RK, McNicholas MMJ, Movson J, Pearlman J, Joffe N (1997) Multiplanar helical CT enterography in patients with Crohn's disease. AJR Am J Roentgenol 169:15451550

6. Hong SS, Kim AH, Buyn JH, Huyng JW, Won HJ, Kim PN, Lee MG, Ha HK (2006) MDCT of small-bowel disease: value of 3D imaging. AJR Am J Roentgenol 187:1212-1221

7. Mulkens TH, Daineffe S, De Wijngaert R, Bellinck P, Leonard A, Smet G, Termote JL (2007) Urinary stone disease: comparison of standard-dose and low-dose with 4D MDCT tube current modulation. AJR Am J Roentgenol 188(2):553-562

8. Tack D, Bohy P, Perlot I, De Maertelaer V, Alkeilani O, Sourtzis S, Gevenois PA (2005) Suspected acute colon diverticulitis: imaging with low-dose unenhanced multi-detector row CT. Radiology 237 (1):189-196

9. Leader JK, Warfel TE, Fuhrman CR et al (2005) Pulmonary nodule detection with low-dose CT of the lung: agreement among radiologists. AJR Am J Roentgenol 185(4):973-978

10. Calabrese E, La Seta F, Buccellato A, Virdone R, Pallotta N, Corazziari E, Cottone M (2005) Crohn's disease: a comparative prospective study of transabdominal ultrasonography, small intestine contrast ultrasonography, and small bowel enema. Inflamm Bowel Dis 11(2):139-145

11. Ochsenkuhn T, Herrmann K, Schoenberg SO, Reiser MF, Goke B, Sackmann M (2004) Crohn disease of the small bowel proximal to the terminal ileum: detection by MR-enteroclysis. Scand J Gastroenterol 39(10):953-960

12. Bodily KD, Fletcher JG, Solem CA, Johnson CD, Fidler JL, Barlow JM, Bruesewitz MR, McCullough CH, Sandborn WJ, Loftus EV, Harmsen WS, Crownhart BS (2006) Crohn disease: mural attenuation and thickness at contrast-enhanced $\mathrm{CT}$ enterography - correlation with endoscopic and histologic findings of inflammation. Radiology 238:505-516

13. Booya F, Fletcher JG, Huprich JE, Barlow JM, Johnson CD, Fidler JL, Solem CA, Sandborn WJ, Loftus EV, Harmsen WS (2006) Active Crohn disease: CT findings and interobserver agreement for enteric phase CT enterography. Radiology 241:787-795

14. Bernstein CN, Boult IF, Greenberg HM, Van der Putten W et al (1997) A prospective randomized study of small bowel enteroclysis vs small bowel follow-through in patients with Crohn's disease. Gastroenterology 113:390-398

15. Ahualli J (2007) The fat halo sign. Radiology 242:945-946 